\title{
The future: will it work?
}

\author{
Gillian Page \\ Pageant Publishing, 1 Weir Gardens, Pershore WA10 1DX, UK
}

It was in 1977 that I first met Jack when he chaired a talk that I was giving on the economics of journal publishing. Since then, we worked together on the book, Journal Publishing and he roped me in for various tasks in association with the Primary Communications Research Centre (PCRC) at Leicester. In spite of all the problems of universities, the chores of university administration and the variety of hats he has worn since then, he seems as ebullient as ever, full of ideas and good humour, tempered with a certain scepticism and an example to us all.

Others contributors have mentioned the wide range of his scholarly interests, but to this must be added the roles he has played in publishing, not simply studying it and as an author, but he has edited books and journals, and, at the PCRC was also a publisher himself.

We do not imagine a future without scientific research; the finalization of science is not on our horizon. Since what is not published is not part of the body of known science, the publishing of scientific research papers seems to have a promising future. The question is in what format and what medium will those papers be published?

It was about twenty years ago that John Senders, one of the earliest proponents and explorers of the electronic journal, pronounced that he had seen the future of scientific publishing and that it did not work. He discovered that if the technology and the systems did not deliver what the people wanted, in the way in which they wanted, and when they wanted it, people would not use them. His project pre-dated the PC, let alone the networked PC. The Internet, the Intranet and the deregulation of telecommunications were a long way off. Downloading and local printing may be slow today, but it was even slower and more costly then.

Now millions of people throughout the world use e-mail and the Web as a matter of routine from homes and offices. This has changed the way we work, get information, communicate with others and react with each other. Will the journal that has hitherto been the main medium of scientific publishing survive these changes? Has the time come to re-evaluate it?

Some believe that new developments have made the journal obsolete now, and that, because of reductions in the numbers of subscribers and increases in prices, the whole system will implode before too long. Alternative models, including the deconstructed journal, are proposed.

A heap of stones is not the same as the building it once was. If we deconstruct a scientific journal we still have the papers it included, but we have lost the journal. What is the difference? The architecture and the cement have gone. Journals are not just collections of papers in a given subject. They have different characters and they enhance the papers they publish in a number of ways. They provide a focus for their subject; and for a community; they present research in the context of other work, an evaluation of current status; at the same time they are a resource for readers, and the archive of their subject. Leading journals give an international view, publishing work not just from one country, let alone a single institution.

Journals have their own communities and their own identities: sometimes they are the centre piece of a society or association. They reflect schools of thought, research programmes and ideas of what are 
the most promising and interesting avenues of exploration. Most human beings gain satisfaction from belonging to groups and being recognised as members of them; books have been written about how the corporation has replaced the tribe in modern society. Having one's first paper published in a leading journal in one's field is a sign that one has been accepted as a member of that community. The scientist who cannot name the journals in his own discipline in which he would prefer to be published must be rare. Each paper gains by association with the rest of the journal.

There may be items other than other original research papers, including editorials, photographs, comments on classic or recent papers, themed issues, reviews, mini-reviews, letters to the editor, fillers of all kinds, book reviews, notices of meetings and so on, at least some of which will be commissioned. Together they and the other contents give a picture of the state of the subject, what is hot and what is not. They reflect the editors and those who advise them: associates, referees, editorial board members, hanging committees and so on.

It is generally agreed that refereeing is necessary, whatever form scientific publishing (as distinct from pre-publication databases) may take. We want an information warehouse, not a car-boot sale. As Jack and his colleagues at the Primary Communications Research Centre showed, refereeing is fallible - but, like democracy, no-one has yet devised a better system. Rejected papers or those whose authors disagree with the revisions requested can be submitted to other journals. Alas, some get into the literature that should not, and may then be cited as authoritative: one study found 299 references to retracted papers of which only 19 mentioned the retraction [1]. This is a serious problem. What can be done about it? Could the defects have been picked up by the referees? What can be done to ensure that retracted papers are not cited as though they are part of the established literature?

Evaluation for publication is not simply a matter of yes or no, pass or fail. Many papers will be returned for revision; many senior scientists will admit that every paper they have written has been improved as a result of the referees' comments. Some editors or referees spend a lot of time working with less experienced authors, or those who have difficulties with the language, helping them to express their ideas more clearly. At the margin, an average publishable paper may be accepted with enthusiasm because it is in a field that they want to develop, or rejected because it does not take the journal in the direction in which the editors want it to go. After acceptance comes the editing. Few papers cannot be improved by a competent copy-editor. Copy-editors check the paper to see that everything is there, clear up ambiguities, reduce duplication, remove howlers and generally improve presentation; they may also check the references. Most authors and probably all readers have cause to be grateful to them.

For their readers, journals are a resource. It seems that most working scientists scan some journals regularly - perhaps something between seven and twelve titles - but certainly not all the journals in their field that are available to them. They are not simply reading research papers relevant to their work. They are seeing what is going on, who is doing what, and where the interesting problems are. They may also want to check that they are being cited where they should be and that their work has been recognised. Indeed, studies suggest that while many people may look at, and get something from, a given paper, few actually read it all through.

Studies of the use of e-journals (such as CORE [2] and the Superjournal project [3]) have found that while scientists are delighted to use the e-version to locate items of interest to them, they then print them out rather than reading on the screen. This is entirely sensible, since reading on the screen is much less efficient (some reckon about one third slower than from paper). It is even more difficult if one is reading in a foreign language. But these studies also found that people want to make notes on what they are reading and that doing so helps absorption and understanding. Apart from that, if you have printed something out, you have captured it - and may then never need to read it at all. These projects also found 
that researchers want what they see and print out to look like the journals they are used to, and to have the same markers as the printed journal: volume, issue, date, page number and so on.

The diversity of journals makes for flexibility. Journal publishing can be done on a small scale, without involving enormous amounts of capital; conventional publishing is not dependent on the availability of large grants. And journals adapt, some faster than others, to new ideas in editing and publishing. Editors can try out new features or experiment with refereeing procedures. Authors are free to choose where to submit their papers.

The system responds to changes in the volume of publishable material. Existing journals can add more pages or more issues as required. If a group feels the development of a new research area or publication delays in existing journals makes a new journal desirable they can set one up. If a journal has too few submissions, it may close or merge with another. But how much should be published? Librarians who opposed new journals or increases in the extent of existing ones on the grounds of cost, welcome electronic publishing because it removes the constraints of a fixed page limit. These are budgetary arguments, but perhaps we should be more concerned with the academic ones, and the economic ones. From an academic point of view, a paper may be unsatisfactory, but would its publication prevent someone else from repeating the work at great expense?

The last point I want to make about print-on-paper scientific journals is that they are the archive of their subject. The presence of multiple copies, spread across libraries throughout the world, makes it difficult to destroy or tamper with the record. Authors cannot use it to rewrite their intellectual history. They continue to be readable, provided that the paper they are on does not deteriorate. Publishers resent the need to deposit copies free for charge in deposit libraries, and it is a financial burden, particularly for small circulation journals. But the system is probably less onerous than requiring publishers to maintain their own electronic archives and certainly more secure than that - or than national electronic archives and mirror sites would be.

So much for the journal model. Other models propose that instead of submitting their papers to a journal, scientists should post them on their own site (scholarly skywriting); have it posted (after some refereeing) on a site run by their university or institution or submit it to an evaluation centre and then, having received a stamp of approval, put it up on a subject focal point. These models are highly debatable and raise many problems, far too many for a paper like this. But there is a fundamental question that has to be asked.

Is there is a large body of scientists who would prefer to post their papers on a site to being published in a leading journal in their field? Will they forgo the contribution made to the final paper by editors, referees and copy-editors? And what about their offprints?

There are of course those who believe in a wholly electronic future. There are those who feel that speed of publication, at least for their work, outweighs all other considerations, including checking and refereeing. Some may have had unhappy experiences with existing journals, their editors, referees, copyeditors, printers, or publishers and want to avoid that again at all costs. We are told some authors care desperately about copyright, though many know very little about it; and publishers are moving towards asking for comprehensive publishing rights rather than copyright assignment So some will switch, but will many follow?

Are e-journals flourishing? Three launched recently by major scientific societies have so far attracted relatively few papers. The American Physical Society Physical Review Special Topics - Accelerators and Beams has published 20 articles in the first six issues of this year; The New Journal of Physics from the Institute of Physics and the Deutsche Physikalishe Gesellshaft has published 17 papers this year, and, to be fair, that is in spite of a charge of US \$500 per paper to the author; Physical Chemical 
Communications from the Royal Society of Chemistry (which also has the backing of the Scholarly Publishing and Academic Resources Coalition of the Association of Research Libraries) published a total of ten papers between 1 October 1998 and 14 June this year. A print-on-paper journal that attracted so few papers would be a cause of great concern to the publisher. If publishers like these, with a stable of major journals, experience of electronic publishing and a large membership from which authors can be drawn are able to attract so few authors, what hope is there for an institutional or subject focused site? Authors need visibility in among their peers in their own discipline. What pressure could a university put on people on short term contracts to publish on the university site rather than in a journal? Where is the attraction of a subject focus site?

Given these problems, those of archiving and the perceived need for something that looks like print on paper, I believe that the journal will continue to be the main medium of publication of scientific research for many years to come. Furthermore, it seems likely that most will for continue to be available in hard copy, though some with e-editions too. E-only journals will attract some authors and may be costeffective in some disciplines. In the developed world, much of the costs of e-publishing can be hidden with the widespread availability of pcs and printers in offices and homes. In the less developed world, the infrastructure is not there, and one can buy an awful lot of print-on-paper journals for the cost of providing the hardware, or even the consumables to the faculty.

If this is right, one must ask why publishers are investing so much time, thought, and hard cash making their journals available online?

The reason is not that in-depth marketing studies have shown an enormous demand for e-access; indeed, as some will confess, many are doing it because other publishers are. Every publisher wants to be able to speak authoritatively about the e-market from first-hand experience. But there are editorial reasons too. Publishers need journals. Societies and other groups looking for a publisher for a journal today want one that offers electronic access, either now or very soon. At the same time, they are responding to pressure from some librarians who are convinced that the future is digital and that publishers who do not make their journals available in e-formats are doomed to extinction.

What seems to be emerging clearly at this stage is that readers welcome electronic access to journals, but they see that as an addition to, rather than a substitute for a paper version. The Superjournal project found that readers still wanted to visit the library and to browse through journals, while they found the electronic version was a quick and easy way of getting hold of a copy of an article, which they would then print out without visiting the library. It also alerted them to articles in journals which they had not previously looked at, and sometimes which were not available in their library.

Librarians, publishers, university administrators, consultants, technical wizards and visionaries can draw marvellous scenarios for the future of academic science publishing, but the Senders problem remains: unless the systems deliver what the users want, when they want it and in the way in which they want it, people will not use them.

Moreover, we want it all - and now, please. Those who have tasted the delights of electronic access will not let us put the electronic genie back in the box, but equally they want to have print-on-paper versions of the same material. If the future is to work, we can expect academic scientific research to be published in journals which are available in both versions.

Ironically, much of the impetus for electronic publishing came from the belief that it would reduce costs. Though many observers are hazy about the real costs of publishing, it is obvious that two versions cost more than one. Who will pay for the additional benefits? It could be either the producer or the consumer of scientific information, but it looks as though the market will demand that the money is found. In real life, the sums are trivial compared with the costs of doing the research, equipping everyone 
with pcs and printers, or running a university. The hope that I have is that monitoring the use of e-journals will demonstrate how much larger is the readership of scientific journals and how much time is spent by scientists on the literature than is currently believed. If so, that will provide the economic justification for more money.

It also looks as though we need not worry about a shortage of projects for informatics research. However, I suspect the real winner will be the person who develops a neat, cheap and efficient printer of downloaded material and manages to receive a sum, however minute, for each page printed.

\section{References}

[1] J.M. Budd, M. Sievert and T.R. Schultz, Phenomena of retraction: reasons for retraction and citations to the publications, Journal of the American Medical Association 280(3) (1988), 296-297.

[2] J. Olsen, Electronic Journal Literature: Implications for Scholars, Mecklermedia, Westport, CT, 1944.

[3] D. Pullinger, The Superjournal Project, Report in preparation. 